LA-UR-01-5836

Approved for public release; distribution is unlimited.

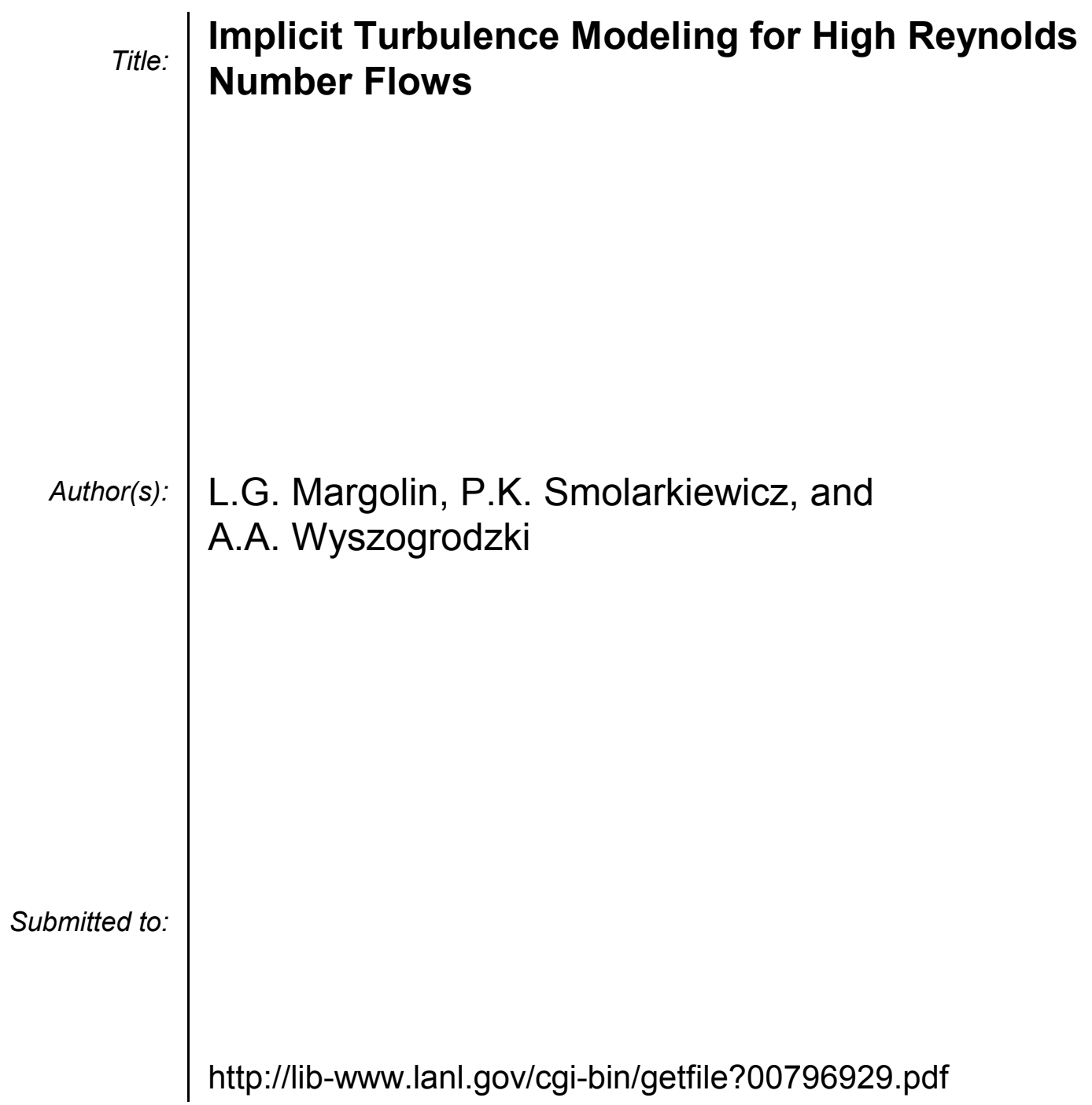

Los Alamos National Laboratory, an affirmative action/equal opportunity employer, is operated by the University of California for the U.S. Department of Energy under contract W-7405-ENG-36. By acceptance of this article, the publisher recognizes that the U.S. Government retains a nonexclusive, royaltyfree license to publish or reproduce the published form of this contribution, or to allow others to do so, for U.S. Government purposes. Los Alamos National Laboratory requests that the publisher identify this article as work performed under the auspices of the U.S. Department of Energy. Los Alamos National Laboratory strongly supports academic freedom and a researcher's right to publish; as an institution, however, the Laboratory does not endorse the viewpoint of a publication or guarantee its technical correctness. 


\title{
Implicit Turbulence Modeling for High Reynolds Number Flows*
}

\author{
L.G. Margolin ${ }^{\dagger} \quad$ P.K. Smolarkiewicz, ${ }^{\ddagger} \quad$ A.A. Wyszogrodzki, ${ }^{\S}$
}

\begin{abstract}
We continue our investigation of the implicit turbulence modeling property of the nonoscillatory finite volume scheme MPDATA. We start by comparing MPDATA simulations of decaying turbulence in a triply periodic cube with analogous pseudospectral studies. In the regime of direct numerical simulation, MPDATA is shown to agree closely with the pseudospectral model. As viscosity is reduced, the two model results diverge. We study the MPDATA results in the inviscid limit, using a combination of mathematical analysis and computational experiment. We validate the inviscid MPDATA results as representing the turbulent flow in the limit of very high Reynolds number.
\end{abstract}

\section{Introduction}

There is a kind of magic about nonoscillatory methods for numerical simulation of complex fluid flows. Beyond the obvious benefits of avoiding unphysical oscillations by preserving positivity and/or monotonicity, and of ensuring nonlinear stability, high-order upwind methods appear to correlate well with the underlying physics,

*This work performed under the auspices of the U.S. Department of Energy by Los Alamos National Laboratory under Contract W-7405-ENG-36. This paper is declared work of the U.S. Government and is not subject to copyright protection in the United States.

${ }^{\dagger}$ Center for Nonlinear Studies, Theoretical Division, Los Alamos National Laboratory, MS B258, Los Alamos, NM 87545, len@lanl.gov

$\ddagger_{\text {Microscale and Mesoscale Meteorology Division, National }}$ Center for Atmospheric Research, Boulder, CO, 80307, smolar@ncar.ucar.edu

$\S$ Institute for Geophysics and Planetary Physics, Earth and Space Sciences Division, Los Alamos National Laboratory, MS C305, Los Alamos, NM 87545, wyszog@kokopelli.lanl.gov; on leave from Institute of Geophysics, Warsaw University, Warsaw, Poland leading to simulations that are more physically realizable.

As a recent and unexpected example of realizability, nonoscillatory methods have demonstrated the ability to simulate turbulent flows without need for explicit subgrid scale models, a property that we refer to as "implicit turbulence modeling." This property has been validated in direct comparisons with experimental data and with high resolution DNS (direct numerical simulation) for a variety of flows, and for a variety of nonoscillatory algorithms (see for example [9], [10], [5], [2]) over the past ten years. In our own research, we have employed the nonoscillatory algorithm MPDATA (for Multidimensional Positive Definite Advection Transport Algorithm; see [15] and references therein) to model allscale meteorological flows including atmospheric boundary layers [8], gravity-wave dynamics [14], and global climate [16].

More recently, the beginnings of a theoretical framework has been proposed for implicit turbulence modeling in [7]. These authors derived a finite-scale (i.e., coarse-grained) version of the point-wise Burgers' equation - a version appropriate for describing the dynamics of finite volumes of (Burgers') fluid. They compared this finite-scale equation to the MPDATA approximation of the point-wise equation, and showed that MPDATA already accounts for the finite-scale effects. Since each computational cell is a finite volume, they rationalized that the success of MPDATA in modeling turbulent flows results from its accurate representation of the coarse-grained equations of motion.

The theory in [7] and the computational examples that support it are suggestive. However there are significant differences between Burgers' and Navier-Stokes equations. Perhaps the most important of these is that the solutions of Burgers' equation are deterministic while those of Navier-Stokes are stochastic. This difference may be readily appreciated by considering simulations at two distinct resolutions. In the case of Burgers' equation the two solutions will be close, while for Navier-Stokes equations it is only the statistics of the solutions that will be close [1].

In this paper, we extrapolate the finite-volume theory of [7] to analyze nonoscillatory simulations of a turbulent flow governed by 3D Navier-Stokes equations. We 
compare MPDATA simulations of decaying turbulence in a triply-periodic cube with the pseudospectral results of Herring and Kerr [3]. For two values of physical viscosity corresponding to direct numerical simulations (DNS) where all dynamical scales are resolved, the MPDATA and the pseudospectral solutions compare closely in all integral measures.

We also compare simulations using zero viscosity. The pseudospectral simulation, in this Euler-equation limit of Navier-Stokes, shows an enstrophy blowup at finite time. The MPDATA simulation tracks the pseudospectral results for a while, but does not show any blowup of enstrophy. The pseudospectral result is unphysical - all physical flows exhibit viscous dissipation at some finite length scale. In contrast, enstrophy in the MPDATA simulation remains uniformly bounded, and the solution appears physically reasonable. However, the question remains whether the MPDATA simulation is the result of a well-posed physical problem, and if so, what this problem is.

To address this question - the central issue of the paper - we analyze the zero-viscosity MPDATA results by combining theoretical arguments with computational experiments. In section III. we derive a relation between the numerical energy spectra at different resolutions. In section IV. we validate this relation computationally. A theoretical consequence is the existence of an asymptotic spectrum in the continuum limit. For zero explicit viscosity, we interpret the asymptotic spectrum as the high Reynolds number (Re) limit of viscous flows [4]. We elaborate, and then summarize our conclusions in section $\mathrm{V}$.

\section{MPDATA}

The simulations presented in this paper all employed the nonoscillatory fluid solver MPDATA. We would emphasize that that implicit turbulence modeling appears to be a property of all nonoscillatory finite volume (NFV) schemes, however MPDATA does have some unique features. MPDATA was developed originally for applications in meteorology. Since it is likely unfamiliar to the aeronautics community, we take this opportunity to summarize some of its features. The interested reader can find a comprehensive description of MPDATA in [15], including both the underlying concepts and the details of implementation.

MPDATA in its basic form is sign preserving, but not monotonicity preserving. For meteorological applications, we have found that sign preservation is often sufficient and leads to a less diffusive solution. MPDATA is fully second-order accurate and conservative. A variety of options have been documented [15] that extend MPDATA to full monotonicity preservation, to third-order accuracy, and to fields that do not preserve sign (of which the most important is momentum).
Unlike most NFV methods, MPDATA is not based on the idea of flux limiting. Instead it is based more directly on upwinding. In practical terms, the algorithm consists of a series of donor cell steps; the first step provides a first-order accurate solution while subsequent steps compensate higher-order errors as identified from a modified equation analysis. One important consequence of this approach is that MPDATA is fully multidimensional - i.e., has no spatial splitting errors - which implies significantly reduced mesh dependence.

MPDATA is a full fluid solver. In analyzing the truncation error of approximations to the momentum equation, one finds error terms that depend on the interaction of the advection with the forcing terms, including the pressure gradient. In implementations of NFV algorithms that treat advection separately from the forcings, this error is uncompensated, reducing the order of accuracy of the solution and potentially leading to oscillations and even instability (see [12]). In MPDATA we compensate this error effectively by integrating the forcing terms along a flow trajectory rather than at a point.

MPDATA is implemented in the 3D program EULAG for simulating rotating, stratified flows in complex geometries, [14][16]. The name EULAG alludes to the capability to solve the fluid equations in either an Eulerian (flux form) or a Lagrangian (advective form) framework. The latter uses a semi-Lagrangian algorithm in which an MPDATA type scheme performs as an interpolation routine. However all the simulations in this paper use the Eulerian framework. EULAG can be run for incompressible or anelastic fluids; in either case, we solve an elliptic equation for pressure using a preconditioned generalized conjugate residual solver [13]. EULAG is fully parallelized using message-passing and runs efficiently on a variety of platforms.

\section{Theory}

In this section, we summarize the theoretical results of [7] and describe an extension to support our analysis.

\section{III.1 Background}

In [7], the authors describe a rationale for implicit turbulence modeling. Their analysis begins by deriving the modified equation for MPDATA applied to 1D Burgers' equation. Among the third-order truncation terms, there appears a nonlinear dispersive term of the form $\Delta x^{2} u_{x} u_{x x}$. The authors then construct the governing equation for a finite volume of Burgers' fluid. These equations are derived from the point equations, but are different due to the nonlinearity of the latter, a fact that has long been appreciated by theorists and modelers studying turbulence. What is unexpected is that a straightforward and justifiable derivation of the finite volume equations leads directly to nonlinearly disper- 
sive terms similar to those in the MPDATA modified equation. The rationale for implicit turbulence modeling then is the more accurate approximation of the finite-volume governing equations by MDPATA (and more generally, by NFV algorithms).

The finite scale Burgers' equation merits further discussion. In particular, one might be tempted to interpret the nonlinear dispersive terms as a model for the effects of the unresolved scales of motion - i.e., as a subgrid scale stress. However a careful examination of the derivation shows that this is not the case, a point that is emphasized by the authors. In fact, the finite scale equation governs the evolution of the volume-averaged velocity independent of the details of the subgrid scale velocity field. In other words, the nonlinear dispersive term regularizes the flow in much the same way that artificial viscosity regularizes shocks in high speed flows (see [11] and [2] for a similar point of view).

There is another, complementary point of view presented in [7]. The finite scale equation can be considered as a model for the measurements (experimental or computational) made by some observer. Experimental devices and simulations both have finite scales of length and time, implying that information about unresolved scales is lost and the measurements do not exactly correspond to "the flow". Since we are in a regime of classical physics, we are not concerned that the measurement process alters the flow, and so we should expect that the measurements accurately reflect the resolved scales. In this sense, the finite scale equation is a better model of the observations than the point equation. One of our principal results in section IV. is to verify that the MPDATA simulations do accurately reproduce the large scales of the turbulence.

\section{III.2 Extensions}

The generalization of the analysis in [7] to 3D NavierStokes is beyond the scope of this paper. Instead, we shall assume that the basic result of the Burgers' equation analysis - that MPDATA estimates the volumeaveraged velocity - remains true for more general equations. This simple and reasonable assumption will allow us to predict the relationship of turbulent energy spectra produced at different resolutions. The verification of this relationship then reinforces our understanding of the performance of MPDATA.

Consider a 1D periodic domain of length $\mathrm{L}$. A measurable velocity $u(x)$ can be expanded in a Fourier series:

$$
u(x)=\sum_{k=0}^{\infty}\left[a_{k} \cos \left(\frac{2 \pi k x}{L}\right)+b_{k} \sin \left(\frac{2 \pi k x}{L}\right)\right] .
$$

Now consider a small segment $[x-\Delta x / 2, x+\Delta x / 2]$. In a 1D simulation, this would represent a computational cell, and $\Delta x / L \equiv 1 / N$ where $N$ is the total number of cells. We now compute the averaged component $\bar{u}$ in this "cell":

$$
\bar{u}(x) \equiv \frac{1}{\Delta x} \int_{x-\Delta x / 2}^{x+\Delta x / 2} u\left(x^{\prime}\right) d x^{\prime} .
$$

An elementary calculation leads to the result:

$$
\begin{aligned}
\bar{u}(x)=\sum_{k=0}^{\infty}\left[a_{k} \cos \left(\frac{2 \pi k x}{L}\right)+\right. \\
\left.b_{k} \sin \left(\frac{2 \pi k x}{L}\right)\right] f\left(\frac{\pi k \Delta x}{L}\right),
\end{aligned}
$$

where the function

$$
f(X) \equiv \frac{\sin X}{X}
$$

attenuates each of the spectral coefficients of the original velocity in a wavenumber dependent fashion. The 1D volume-averaged energy associated with $\bar{u}$ can now be written as

$$
\begin{aligned}
\bar{E}(k) & =\left(a_{k}^{2}+b_{k}^{2}\right) f^{2}\left(\frac{\pi k \Delta x}{L}\right) \\
& =E(k) f^{2}\left(\frac{\pi k \Delta x}{L}\right) .
\end{aligned}
$$

These calculations can be easily extended to 3D; however using the anticipated isotropy of the velocity field, the above result can be applied directly in our simulations. In fact we have calculated the energy spectra shown later in Figs. 2, 3, and 4, by averaging the 1D spectra calculated in each of the three coordinate directions.

Let us now identify $\overline{\mathbf{u}}$ as the discrete values of the velocity components in an MPDATA simulation. Equation (5) can be used to estimate the underlying energy spectrum in part - up to the finite wavenumber determined by the resolution of the simulation. We term

$$
E(k) \equiv \frac{\bar{E}(k)}{f^{2}(\pi k / N)}
$$

the asymptotic spectrum. Furthermore, since the simulated spectrum at each resolution has the same asymptotic spectrum, we can relate the energy spectral coefficients of two simulations at resolutions $N_{1}$ and $N_{2}$

$$
\frac{\bar{E}_{1}(k)}{f^{2}\left(\pi k / N_{1}\right)}=\frac{\bar{E}_{2}(k)}{f^{2}\left(\pi k / N_{2}\right)},
$$

where this relation holds up to the largest wavenumber of the more coarsely resolved simulation.

We close this section with these remarks about our result. First, the derivation of eqs. (6) and (7) does not depend on the form of the governing equations, and are not specific to Navier-Stokes. Second, these equations allow us to estimate an asymptotic spectrum, given a simulated spectrum at finite resolution, but do not predict any universal form for this spectrum. Third, for small values of its argument, eq. (6) implies convergence of the simulated spectra to the asymptotic spectrum as $1 / N^{2}$. 


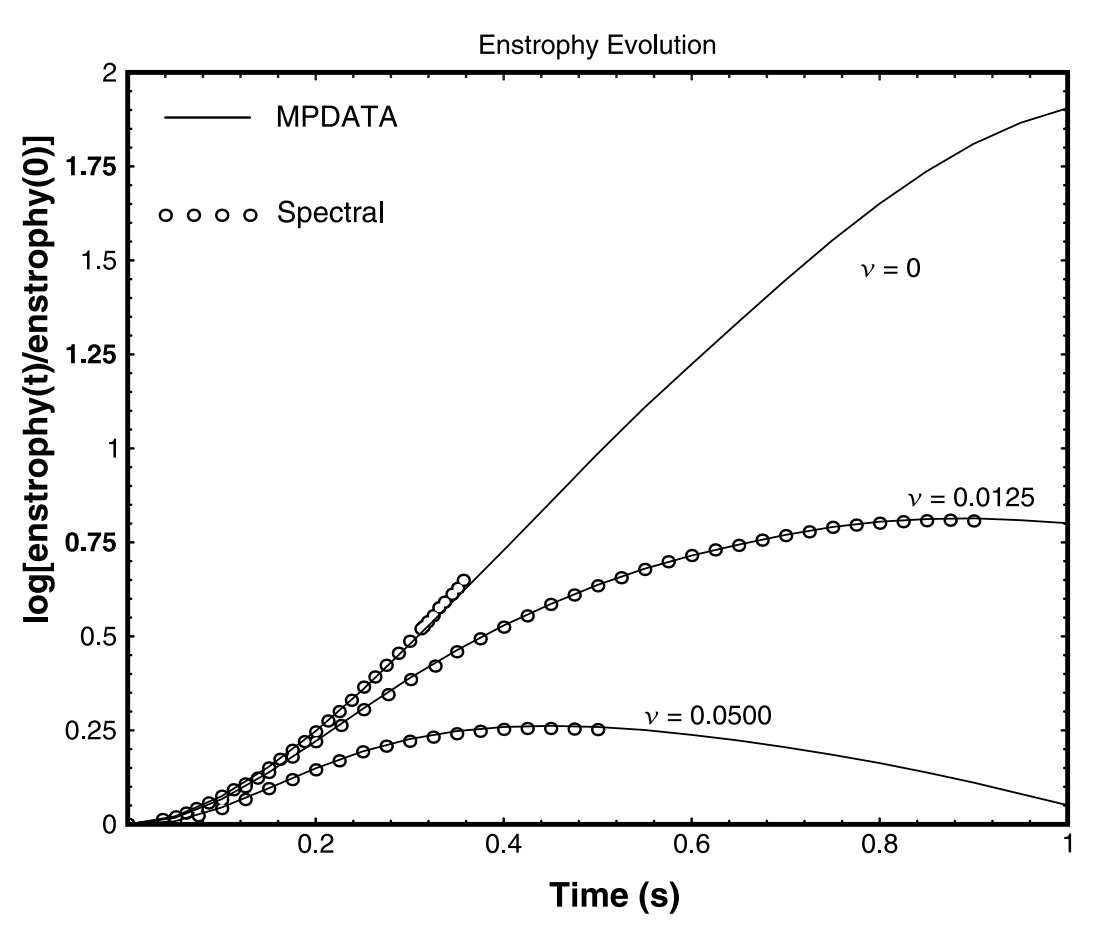

Figure 1: Enstrophy history in pseudospectral and MPDATA simulations of isotropic decaying turbulence.

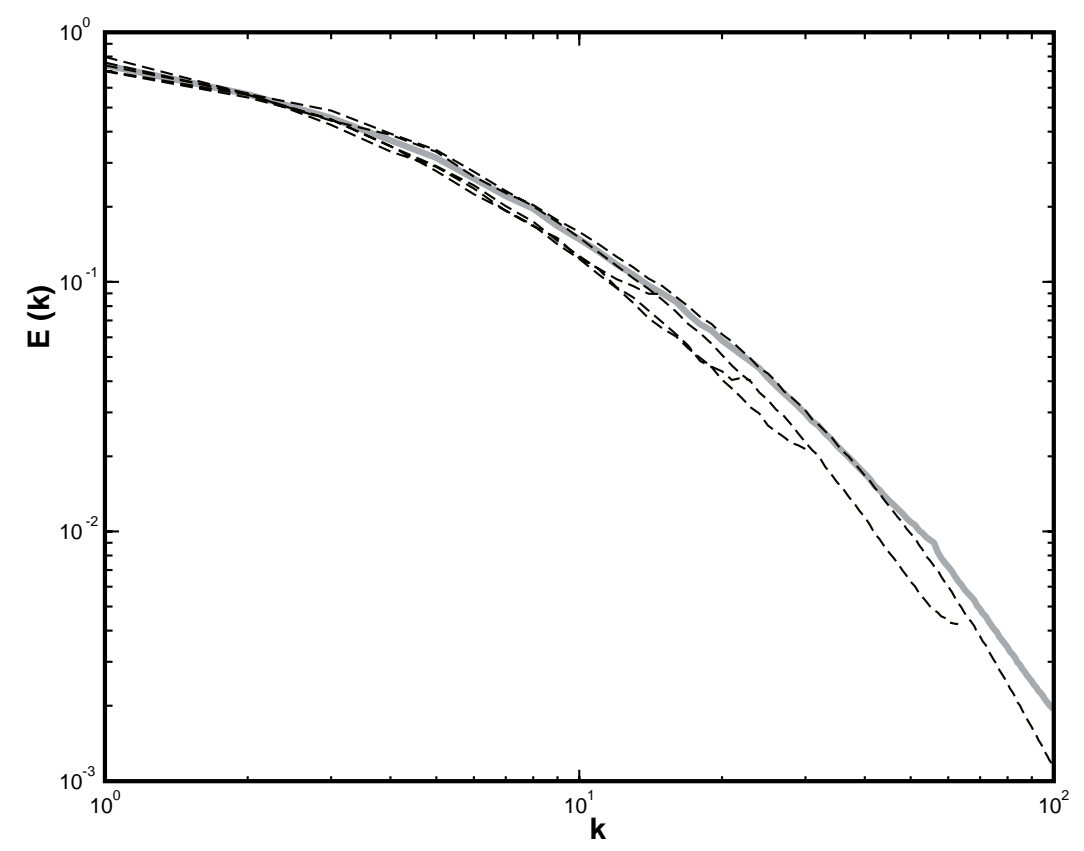

Figure 2: MPDATA simulated spectra for zero viscosity at differing resolutions $-32^{3}, 48^{3}, 64^{3}, 128^{3}, 256^{3}$ 


\section{Analysis and Results}

We will analyze the simulations of decaying turbulence of a homogenous incompressible fluid in a triplyperiodic cube - a generic problem in turbulence studies. The assumed homogeneity of the thermodynamics and the lack of near-wall effects, focus attention on the modeling of the convective derivatives $\mathbf{u} \nabla \mathbf{u}$ in the momentum equation. Our NFV experiments with MPDATA will mirror the $256^{3}$ DNS and inviscid pseudospectral simulations of Herring and Kerr [3].

Figure 1 displays the numerical data for the evolution of enstrophy for three values of viscosity, $\nu=0.0500$, $\nu=0.0125$, and $\nu=0 \mathrm{~m}^{2} \mathrm{~s}^{-1}$ (as indicated). Solid lines are for MPDATA experiments, while the data from [3] are marked as circles. One striking result in Fig. 1 is the remarkable agreement of the NFV and the pseudospectral solutions for DNS $(\nu>0)$. This agreement is maintained uniformly for all flow characteristics, including spectra (see [3], for other diagnostics). Traditionally, pseudospectral methods are valued for their accuracy and have been considered the tool of choice to study turbulent flows. Since all convergent methods become accurate as the flow is fully resolved, one may wonder whether this agreement is due to excess resolution. This is definitely not the case for $\nu=0.0125$ where the Kolmogorov scale is about one grid-interval (Kerr, personal communication) and the energy dissipation is marginally resolved.

The $\nu=0$ results expose the essential difference between the pseudospectral and MPDATA approaches. Without viscous dissipation, enstrophy blowup occurred in the pseudospectral solution. The rapid growth of enstrophy was accompanied by an energy buildup at the highest wave numbers; the spectral calculations became unstable, and were terminated after $\sim 0.35 \mathrm{~s}$ [3]. Up to this point, the spectral and MPDATA results agree closely. Beyond this point, MPDATA continues to produce a plausible solution. However, it is not clear whether the MPDATA simulation is physically realizable.

Based on the large eddy simulations (LES) of boundary layers in [8], we interpret the $\nu=0$ MPDATA result as the finite-scale representation of the $R e \rightarrow \infty$ limit of viscous flows. This contrasts with the pseudospectral result, which at $\nu=0$ represents the solution of the point-wise Euler equations where finite-scale effects have been already discarded. To substantiate our interpretation, we have compiled two convergence studies of the inviscid solution. The first study shows the convergence of the energy spectra as a function of resolution for zero viscosity. The second study shows the convergence of the energy spectra as a function of viscosity.

Figure 2 compares the energy spectra from MPDATA simulations at $\nu=0$ for a series of resolutions $32^{3}, 48^{3}$, $64^{3}, 128^{3}$, and $256^{3}$, as indicated. The spectra follow each other closely at the large scales of motion, but tend to separate at the smaller scales. This separation is predicted theoretically in section III., which also implies the existence of the unique asymptotic spectrum (eq. 6). Figure 3 displays the asymptotic spectra generated from those shown in Fig. 2. In the absence of truncation errors, all asymptotic spectra should coincide for the resolved scales. Except for the very highest wavenumbers, where the theory of [7] does not apply, ${ }^{1}$ the discrepancies of the asymptotic spectra are small compared to the separation of the simulated spectra (Fig. 2). The latter indicates that the MPDATA results should be interpreted as a finite-resolution projection of the continuum limit. Note that the solid line in Fig. 2, showing the asymptotic spectrum averaged over all five resolutions, estimates the converged limit of the simulated spectra.

As remarked earlier, all physical flows have viscous dissipation. Although the viscous scale lengths may be small compared to finite resolution of a numerical model, they never vanish in the continuum limit. To assess the effects of small but finite viscosity we show in Fig. 4 the convergence of the asymptotic energy spectra as $\nu \rightarrow 0$; these spectra are estimated from MPDATA simulations at $256^{3}$ resolution. The results shown demonstrate that the viscous spectra converge uniformly to the inviscid limit as $\nu \rightarrow 0$. Taken together, our computations substantiate our contention that the inviscid MPDATA runs are finite-scale representation of the $R e \rightarrow \infty$ limit of viscous flows.

\section{Summary and Conclusions}

In this paper, we have continued our study of the use of the nonoscillatory finite volume scheme MPDATA to simulate turbulent flow without need for any explicit subgrid model. We refer to this property as implicit turbulence modeling. We began by comparing simulations of a turbulent flow using MPDATA to a pseudospectral simulation. When the physical viscosity is large enough so that all dynamical scales are well resolved, we have shown that MPDATA accurately reproduces the flow predicted by the pseudospectral model, thus validating the utility of this NFV scheme for direct numerical simulation.

In the limit of vanishing physical viscosity, the pseudospectral model predicts a enstrophy blowup in finite time, and the cessation of meaningful calculation after this point. In contrast, MPDATA does not predict any blowup of enstrophy and continues to produce plausible solutions. Our principal result in this paper has been to analyze these solutions, to demonstrate that they are in

\footnotetext{
${ }^{1}$ The simulated and asymptotic spectra show the well-known bottleneck effect [6] which only affects the highest resolved wave numbers. These data have been ignored while constructing the average asymptotic spectrum (solid line in Fig. 2).
} 


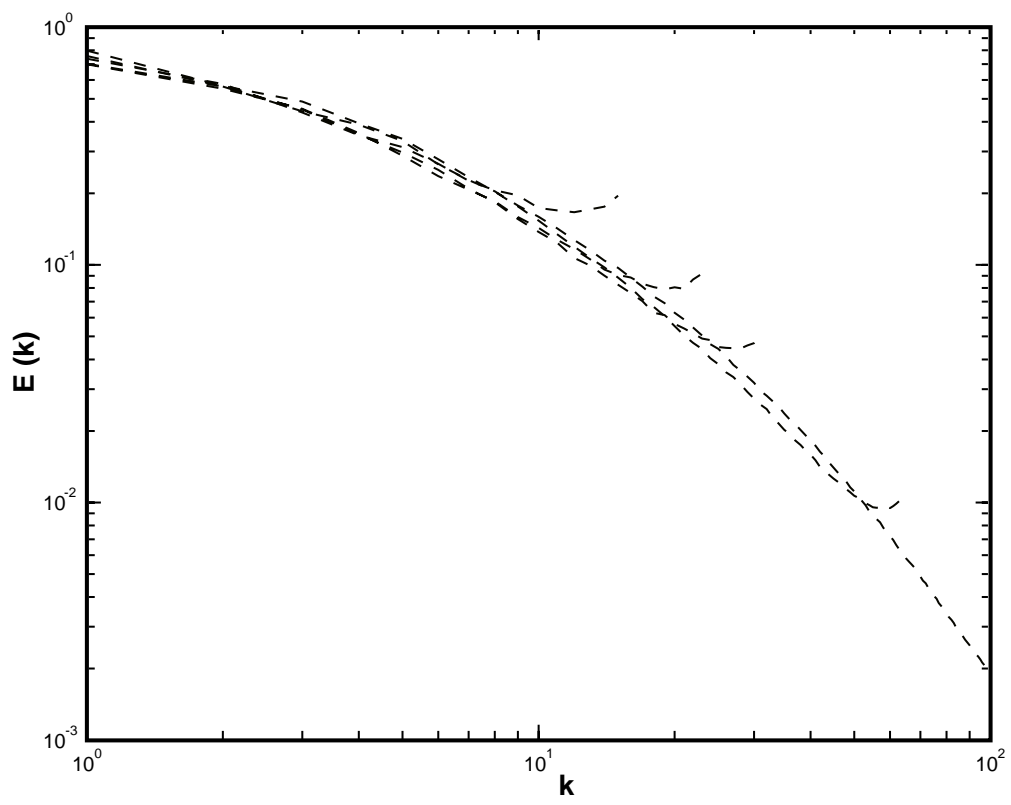

Figure 3: Asymptotic spectra for zero viscosity estimated from simulated spectra in Fig. 2

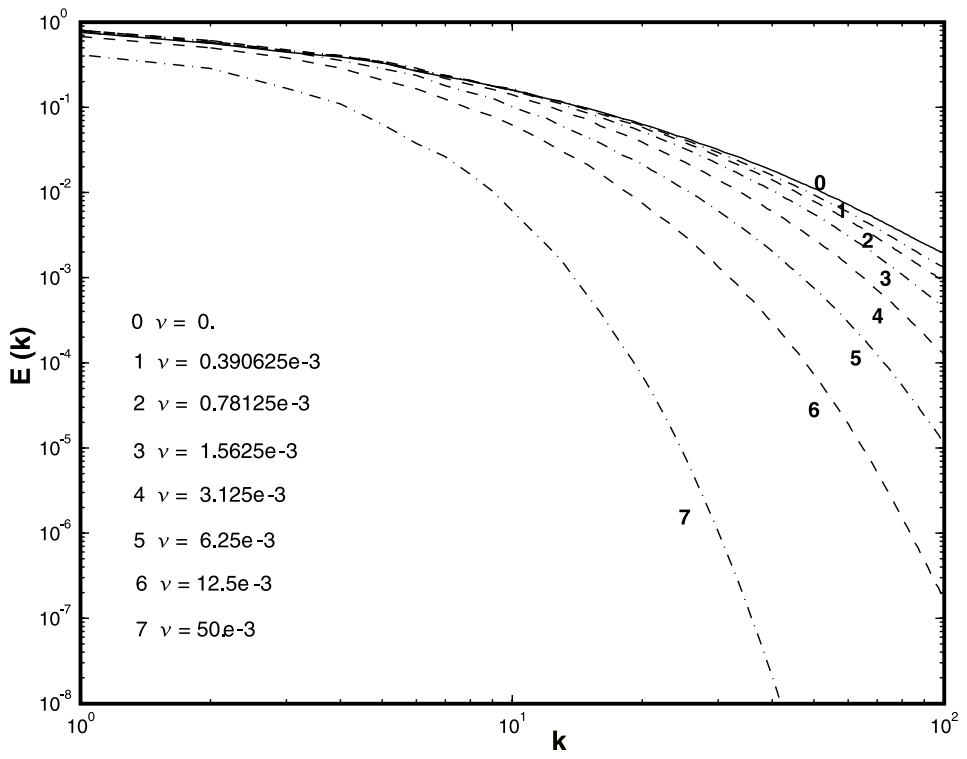

Figure 4: Asymptotic spectra for finite viscosities. 
fact realizable, and to identify them with a physical problem. Our demonstration relies on mathematical analysis and a computational study of convergence in resolution and viscosity.

Our analytic result is derived in section III. and represents a relationship between the energy spectra generated in simulations at different resolutions. The results assume that the numerical algorithm MPDATA accurately estimates the volume-averaged velocity; this result has been demonstrated rigorously for the Burgers' equation in [7]. Our analytic result further implies the existence of an "asymptotic" spectrum, representing the continuum limit, and verifies that at fixed wavenumber the convergence rate goes like the inverse square of the resolution.

Our numerical results in section IV. validate the analytic relations, and confirm the existence of a unique asymptotic energy spectrum. We further showed a computational study of the convergence of the asymptotic viscous spectra to the inviscid spectrum. This leads to our main conclusion, that the MPDATA simulations with zero explicit viscosity correctly model turbulent flow in a high Reynolds number limit.

Throughout this paper, we have emphasized that numerical simulation and physical observation are both carried out at finite scales. In creating a model for numerical simulation of high Reynolds number flow, one is effectively considering the limit as two different length scales become small. The first is the viscous scale, sometimes termed the Kolmogorov scale. The second is the scale of nonlinear effects (see section III.) which is the scale of observation or, in the case of simulation, the size of the computational cell. The latter is never negligible in a simulation — so the Euler equations cannot be an adequate model for numerical simulation of high Reynolds number flow - and the finite scale effects must be taken into account. Based on the results presented, we surmise that MPDATA (and likely other NFV algorithms) accurately models coarse-grained solutions of 3D Navier-Stokes equations, and appears to be a valuable tool for large eddy simulation free of explicit subgrid-scale parameterization.

Acknowledgements: Helpful discussions with Shiyi Chen and Robert Kerr are gratefully acknowledged. Los Alamos National Laboratory is operated by the University of California for the U.S. Department of Energy. National Center for Atmospheric Research is sponsored by the National Science Foundation. This work has been supported in part by the Department of Energy "Climate Change Prediction Program" research initiative.

\section{References}

[1] U. Frisch, "Turbulence, The Legacy of A.N. Kolmogorov" Cambridge University Press (Cam- bridge, UK), 1995.

[2] C. Fureby, F.F. Grinstein, "Monotonically integrated large eddy simulation of free shear flows," AIAA Journal, 37 544-556 (1999).

[3] J.R. Herring, R.M. Kerr, "Development of enstrophy and spectra in numerical turbulence," Phys. Fluids A, 5 2792-2798 (1993).

[4] A.N. Kolmogorov, "The local structure of turbulence in incompressible viscous fluid for very large Reynolds numbers," Dokl. Akad. Nauk SSSR, 30, 301-305 (1941).

[5] P.F. Linden, J.M. Redondo, D.L. Youngs, "Molecular mixing in Rayleigh-Taylor instability," J. Fluid Mech. 265 97-124 (1994).

[6] D. Lohse, A. Miller-Groeling, "Bottleneck effects in turbulence: Scaling phenomena in r- versus pspace," Phys. Rev. Lett., 74, 1747-1750 (1995).

[7] L.G. Margolin, W.J. Rider, "A rationale for implicit turbulence modeling," Proceeding, ECCOMAS Computational Fluid Dynamics Conference 2001, Swansea, Wales, UK, 4-7 September, 2001.

[8] L.G. Margolin, P.K. Smolarkiewicz and Z. Sorbjan, "Large eddy simulations of convective boundary layers using nonoscillatory differencing," Physica D 133 390-397 (1999).

[9] E.S. Oran, J.P. Boris, " Computing turbulent shear flows - a convenient conspiracy," Computers in Physics 7 523-533 (1993).

[10] D.H. Porter, A. Pouquet, P.R. Woodward, "Kolmogorov-like spectra in decaying threedimensional supersonic flows," Phys. Fluids 6 2133-2142 (1994).

[11] J. Smagorinsky, "Some historical remarks on the use of nonlinear viscosities," In Large Eddy Simulation of Complex Engineering and Geophysical Flows, 3-36, Cambridge University Press (1993).

[12] P.K. Smolarkiewicz, L.G. Margolin, "On Forwardin-time differencing for fluids: Extension to a curvilinear framework," Mon. Wea. Rev. 121 1847-1859 (1993).

[13] P.K. Smolarkiewicz, L.G. Margolin, "Variational solver for elliptic problems in atmospheric flows," Appl. Math. \& Comp. Sci. 4 527-551 (1994).

[14] P.K. Smolarkiewicz, L.G. Margolin, "On forwardin-time differencing for fluids; an Eulerian/semiLagrangian nonhydrostatic model for stratified flows," Atmos.-Ocean Special, 35 127-152 (1997). 
[15] P.K. Smolarkiewicz, L.G. Margolin, "MPDATA: a finite difference solver for geophysical flows," J. Comput. Phys. 140 459-480 (1998).

[16] P.K. Smolarkiewicz, L.G. Margolin, A.A. Wyszogrodzki "A class of nonhydrostatic global models," J. Atmos. Sci. 58 349-364 (2001). 